\title{
MRI evaluation of anterolateral ligament tears in knee injury with anterior cruciate ligament rupture
}

\author{
Aman Hooda ${ }^{1}$ \\ Mandeep Singh Dhillon ${ }^{1}$ \\ Sharad Prabhakar ${ }^{1}$ \\ Mahesh Prakash ${ }^{2}$ \\ Rakesh John ${ }^{1}$ \\ Himanshu Kanwat ${ }^{1}$
}

1 Department of Orthopedics, Post Graduate Institute of Medical Education and Research (PGIMER), Sector 12, Chandigarh, India 160012

2 Department of Radiology, Post Graduate Institute of Medical Education and Research (PGIMER), Sector 12, Chandigarh, India

Corresponding author:

Mandeep Singh Dhillon

Department of Orthopedics

Post Graduate Institute of Medical Education

and Research (PGIMER)

Sector 12

160012 Chandigarh, India

E-mail:drdhillon@gmail.com

\section{Summary}

Introduction: To evaluate the incidence of anterolateral ligament (ALL) tear in acute knee injury and its association with anterior cruciate ligament tear (ACL).

Methods: Forty patients with isolated early ACL tears were prospectively reviewed under 3T-MRI evaluation to identify ALL tears. This was correlated with trauma mechanisms and degree of knee instability. Patients less than 18, or more than $\mathbf{5 0}$ years of age, and those with posterolateral corner injury or LCL instability were excluded, which left 31 patients (30 males: 1 female; mean age: 28.6 years) for the final evaluation.

Results: The ALL was visualized completely on the MRI in all 31 patients. ALL had mid substance tear in 19 knees (61.3\%), proximal femoral attachment in eight knees (25.8\%), at the tibial end in 6 knees $(19.3 \%)$ and was seen torn at both proximal and distal ends in five knees (16.1\%). Clinical correlation revealed higher number of giving way episodes, more functional loss of activities and higher grades of pivot shift test in cases with ALL tear along with ACL tear as compared with patients who had ACL deficiency without concomitant ALL tear ( $p$ value $<0.05$ ).

Conclusion: The ALL can be identified in all cases with 3T-MRI. Tears are clearly seen and may be classified according to location. We found no correlation of ALL tears with injury mechanism; however ACL deficient knees with concomitant ALL tear on MRI had more functional impairment and instability.

Level of evidence: II b.

KEY WORDS: anterolateral ligament (ALL), knee injury, ACL deficient knee, 3T MRI, knee instability.

\section{Introduction}

Sporadic reports in the past have suggested the presence of a ligamentous structure which connects the femur with the tibia on the anterolateral aspect of the knee. First description dates back to the late $19^{\text {th }}$ century and a correlation with Segond's fracture was presumed $^{1}$. Subsequently, over the next century, the concept of anterolateral instability of knee was propagated. Due to the absence of a clear anatomic description, this inexplicable structure never accomplished wide acceptance in orthopaedic literature.

Recently, however, the anterolateral ligament (ALL) has been recognised as a well-defined ligamentous structure on the anterolateral aspect of the human knee. Cadaveric studies have demonstrated that the $A L L$ originates from the lateral femoral epicondyle of femur, with some fibres integrating into the proximal part of the lateral collateral ligament ${ }^{2,3}$. The ALL further follows an oblique intra-capsular course demonstrating firm attachments to the lateral meniscus (LM). The ALL inserts on to the proximal aspect of the anterolateral tibia midway between the fibular head and the Gerdy's tubercle ${ }^{4,5}$. Current evidence postulates that the ALL functions primarily as an essential internal rotatory stabilizer of the knee, between 30 to $90^{\circ}$ of flexion ${ }^{3}$. With the pivot shift phenomenon basically consisting of a coupled action of anterior subluxation of tibia and excessive internal rotation of tibia, it has been subsequently shown that an injury to the ALL forms a pre-requisite for the occurrence of a high-grade pivot shift test in the anterior cruciate ligament $(A C L)$ deficient knee ${ }^{6}$.

Previous studies which utilised a 1.5 Tesla MRI to 
identify the ALL have been inconsistent ${ }^{7}$. Visualisation of thin structure resembling the ALL was best seen in coronal T2 weighted images or Proton density planes $^{8}$ (PD). ALL divides into 3 parts: the femoral, meniscal and tibial components; this division was identified partially but not as a whole in 1.5T MRI. Recent studies have demonstrated an improved visualisation of ALL with a 3T MRI and all three components of the ligament can be identified separately.

Despite the clinical interest in ALL injury associated instability, the anatomy and function of the structures over the anterolateral aspect of the knee have not been clearly defined. The purpose of our study was to identify the ALL in the knees of Indian patients on $M R I$ and to characterize its association with ACL injury.

\section{Materials and methods}

This study was designed as a prospective cohort study and was conducted between July 2015 and March 2016 at the Postgraduate Institute of Medical Education and Research (PGIMER), Chandigarh, India. The study was cleared by the institutional ethics committee. The study has been conducted ethically according to set international standards and meets the ethical standards of the journal ${ }^{9}$.

All patients aged 18-50 years presenting with ACL injury within 6 months of primary trauma ${ }^{10}$ were included after clinical and radiological confirmation on MRI. An informed, written consent was a necessary prerequisite for all subjects. Those patients undergoing revision $A C L$ reconstruction, suffering from knee injuries potentially affecting imaging of the knee's lateral compartment (i.e. injury of ligamentous posterolateral corner injury including the lateral collateral ligament), patients showing signs of osteoarthritis i.e. Kellgren-Lawrence grade II inflammatory arthritis or hemophilia, and patients with a hardware incompatible with MRI were excluded.

The patients were examined clinically and routine radiographs of the knee were obtained with special emphasis on identifying Segond's fracture. All patients underwent routine MRI sequencing using dedicated knee coil on a 3T Siemens MRI machine including PD fat suppressed (SET) imaging in axial, coronal and saggital planes, PD non-fat SET imaging in coronal plane and T1-weighted sequence in coronal and axial planes. Thin sections in the coronal fat supressed sequences were obtained to evaluate the $A L L^{10}$. The ALL was found to be located 2 to 3 slices anterior to the LCL consistently. Any discontinuity of the ALL or an altered signal change was classified as a tear and was documented.

The data was evaluated by one senior musculoskeletal radiologist and two orthopaedic surgeons independently. On the basis of MRI results, the patient were divided into two groups; first group included subjects with an ALL tear along with an ACL tear and second group included subjects with ACL tear without an as- sociated ALL tear. The ALL tears were further subclassified into femoral component tear, tibial component tear and complete tears. The physical examination findings like number of giving way episodes, functional limitation of activities and grades of pivot shift test ${ }^{11}$ were then correlated with the MRI findings.

\section{Statistical analysis}

The variables under study were measured as categories. The association between variables was determined for statistical significance by using parametric tests (Chi square test/Fischer's exact test). A p-value less than 0.05 was considered statistically significant. The statistical analysis was done using the software IBM-SPSS (Statistical Package for Social Sciences) version 20.

\section{Results}

We evaluated 40 patients with ACL deficiency presenting within 6 months injury over a period of 8 months. Nine knees were excluded out of which 3 knees had no ACL injury on MRI, 3 knees had an associated PLC injury and 3 knees had an associated LCL injury. 31 subjects (30 males, 1 female), mean age was $28.7 \pm 8.67$ years (range: 18 to 48 years) were studied. The mean duration of injury is 3.8 months \pm 1.38 (min 1 month-max 6 months). The patients were divided into two groups according to the mode of injury i.e. 11 patients had a contact injury $(35 \%)$ and 20 patients had a non-contact injury $(65 \%)$. On taking a detailed history, it was observed that 11 patients had reported more than five episodes of giving way, eight patients had reported two to four episodes of giving way and eight patients had reported a single episode of giving way since primary injury; in four patients no episodes of giving way were noted.

For assessing functional limitation of activities, the patients were specifically questioned as below: "What is the highest level of activity you can perform without significant giving way in your knee?". It was noted that eight patients $(25.8 \%)$ were able to perform strenuous activities like heavy physical work, four patients $(12.9 \%)$ were able to perform moderate activities like running and jogging, ten patients (32.2\%) were able to perform light activities like walking, nine patients $(29 \%)$ were unable to perform any of the above activities without giving way. However no patient could go back to original activities or play a sport after initial trauma. On clinical examination, anterior drawer test, Lachman's test and pivot shift tests were positive in all 31 cases. On further grading the pivot shift test, 12 patients (38.7\%) had grade 1 pivot shift phenomenon (i.e. gentle glide), three patients $(9.68 \%)$ had a grade 2 pivot shift phenomenon (i.e. sudden jerk) and 16 patients (51.6\%) had grade 3 pivot shift phenomenon (i.e. clunk). 


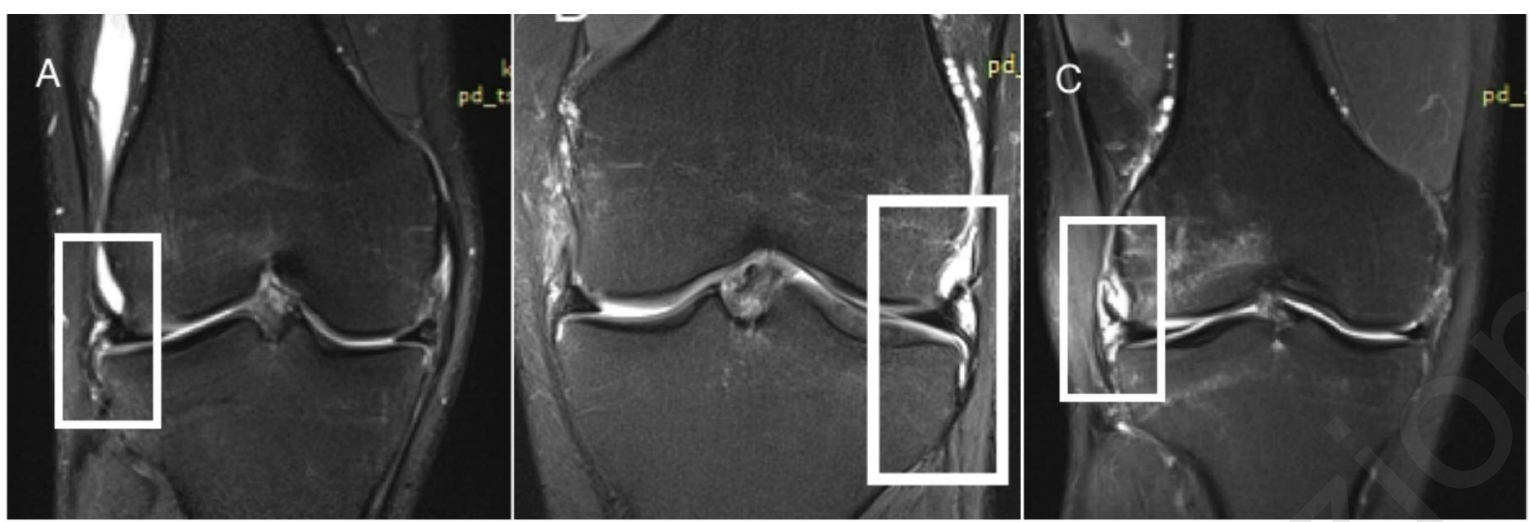

Figure 1. MRI images above show the coronal T2 fat-suppressed cuts [A. Intact ALL; B. ALL tear (femoral component) and C. complete tear of ALL (both femoral and tibial components)].

All 31 knees were subjected to MRI (3T) and we were able to identify the ALL in all cases along with an ACL tear. In 12 knees (38.7\%), the ALL was noted to be in continuity. The inter-observer reliability is $90.3 \%$ between two observers. On detailed evaluation of $\mathrm{MRI}$, it was found that mid substance tear of ALL was present in 19 knees out of 31 (61.3\%), ALL tear at the femoral component in eight knees $(25.8 \%)$, tear at the tibial component in six knees (19.3\%) and complete tear of both tibial and femoral component was observed in five knees (16.1\%) (Fig. 1).

The MRI findings of ALL tear were correlated with the mode of injury, number of giving way episodes, functional limitation of activities and grades of pivot shift test. Out of 19 patients with ALL tear on MRI, six patients sustained a contact injury (31.6\%) whereas 13 patients sustained a non-contact injury (68.4\%). In 12 ACL deficient patients with no ALL tear on MRI, five patients had contact injury $(41.7 \%)$ and seven patients had non-contact injury (58.3\%). It was observed that the mechanism of injury had no significant correlation with the incidence of ALL tear ( $p$ value $>0.05$ ).

The functional limitation of activities was then correlated with MRI confirmed ALL tear cases. Nine patients $(47.4 \%)$ were unable to perform any daily activities due to giving way of knee and were using brace; 10 patients $(52.6 \%)$ were able to do light activities only (like walking, house work or yard work). In $12 \mathrm{ACL}$ deficient patients with no ALL tear, four patients (33.3\%) were able to do moderate activities like running or jogging and eight patients (66.6\%) were able to perform strenuous activities like heavy manual labor. After statistical analysis using Pearson chisquare test, significant ( $p$-value $<0.05$ ) association of ALL tear on MRI with functional loss could be determined.

It was also found that eight patients with MRI-proven ALL tears had two to four episodes of giving way $(42.1 \%)$ whereas 11 patients had more than five episodes of giving way since the original injury (57.9\%). In cases with no ALL tear on MRI, eight patients reported less than two giving way episodes $(66.7 \%)$ and four patients reported no giving way episodes (33.3\%). It was found that patients with ALL tears were having more episodes of giving way as compared with patients who had no ALL tear ( $p$ value <.05).

After correlating ALL finding on MRI with pivot shift grading, 16 out of 19 patients with ALL tear had a grade 3 pivot shift $(51.6 \%)$ and three patients had a grade 2 pivot shift $(9.7 \%)$. In the 12 patients without an ALL tear on MRI, only a grade 1 pivot shift phenomenon was demonstrated. It was observed that patients with ALL tear demonstrated higher grades of pivot shift phenomenon as compared to patients with no ALL injury, who showed low grade of pivot shift phenomenon; this association was statistically significant ( $p$ - value $<0.05$ ) (Tab. I).

Table I. The association of patients showing presence or absence of ALL tear on MRI with grades of pivot shift test.

\begin{tabular}{lllllll}
\hline & & \multicolumn{2}{c}{ Pivot shift grading } & \multicolumn{2}{c}{ Total } \\
& & & One & Two & Three & \\
\hline MRI(3T) & ALL Tear & Patients & 0 & 3 & 16 & 19 \\
& & Percentage & $0 \%$ & $15.8 \%$ & $84.2 \%$ & $100.0 \%$ \\
& No Tear & Patients & 12 & 0 & 0 & 12 \\
& & Percentage & $100.0 \%$ & $0 \%$ & $0 \%$ & $100.0 \%$ \\
\hline
\end{tabular}




\section{Discussion}

The precise anatomy of ALL has been delineated very recently ${ }^{5}$. Evaluation of ligamentous structures around the knee is generally considered effective with $\mathrm{MRI}$, but remains challenging because of the anatomical variations and the thinness of these structures $2,4,5$.

Since 2013 , several cadaveric studies ${ }^{2,5}$ have given detailed descriptions of ALL. Caterine et al. ${ }^{12} \mathrm{com}-$ pared both the dissection and histological analysis with appearance of ALL on MRI in 10 cadaveric knees and found a strong correlation. Subsequently, high detection rates of $A L L$ on $1.5 \mathrm{~T} \mathrm{MRI}^{8}$ have been reported. However, till date there is only one study in the literature, which identifies the ALL in $100 \%$ knees on a $1.5 \mathrm{~T} \mathrm{MRI}^{10}$. That is why we chose $3 \mathrm{~T} \mathrm{MRI}$ in all 31 knees especially on coronal T2 fat-suppressed image sequences which demonstrated the intact and torn ALL.

In injured patient population there has been controversy about the presence or absence or even the significance of the ALL. Thus was probably due to a limited understanding of the pertinent anatomy and inadequate radiological imaging i.e. use of lower resolution MRI, and improperly planned cuts. Higher detection rates on MRI were also reported by Helito et al. ${ }^{8}$, who demonstrated the ALL in $97.8 \%$ of uninjured knees and Kosy et al. who identified ALL in 94\% partially and in $57 \%$ cases fully ${ }^{13}$. In contrast, Taneja et al. did a study of 70 consecutively performed MRIs and could demonstrate the ALL (fully or partially) in only $51 \%$ cases $^{14}$. All the above MRI studies for the identification of ALL were done on 1.5 T MRI machine.

It is important to note that the ALL is a ligamentous structure with a slightly oblique course over the anterolateral aspect of the knee; the standard coronal MRI reconstructions of the knee rarely depict the complete ALL on a single slice ${ }^{13,14}$. However, in some knees where the reconstructed coronal field was (accidentally) inclined, a full-length ALL could be visualized on a single slice from its origin on the lateral femoral epicondyle to its insertion on the tibia ${ }^{13}$. In our study, we were able to demonstrate the ALL on a single slice in few cases but typically the ALL was best visualized on the coronal slices in T2 fat suppressed sequences, taken two or three individual slices anterior to the LCL. In our study, all 31 patients underwent routine MRI sequences including PD fat suppressed (SET) imaging in axial, coronal and saggital plane, PD non-fat SET imaging in coronal plane and T1 weighted sequence in coronal and axial images with no special position of leg. The ALL was visualized in coronal planes T2 fat-suppressed image sequences, but could not be visualized in axial and saggital planes ${ }^{15}$. We specifically planned our study with a higher magnetic strength MRI machine (3T) as 3T MRI has a high signal to noise ratio and shows a better resolution of images. By focussing on coronal T2 fat-suppressed images of ACL deficient knees, we were able to identify the torn ALL in the form of a discontinuity of the ligament or an altered signal change (19 out of 31 knees) (61.3\%). In all other intact ALL was clearly seen. Additionally, on detailed evaluation of these 3T MRI scans, we were able to classify the ALL tear based on the location of the tear (i.e. femoral component or tibial component or both). All MRI scans were done on the same MRI machine to maintain uniformity; images were assessed by one musculoskeletal radiologist (MP) and two orthopedic surgeons independently (AH and SP) and any dispute in the findings were settled after consultation with the senior-most orthopaedic surgeon (MSD).

None of the previous studies have correlated the history and examination findings of the patients with the MRI findings of ALL clearly. Only two studies in the literature support the hypothesis that ALL is a rotational stabiliser of knee ${ }^{6,16}$. Zein ${ }^{6}$ demonstrated the tightening of ALL during internal rotation of tibia arthroscopically. Chahla et al. ${ }^{16}$ compared the rotational stability of knee with pivot shift test to pre-operative state after reconstruction of ALL and found significant improvement in stability. These two studies in the literature support the view that ALL has an important role in pivot shift phenomenon, and underline the importance of identifying injury to this structure.

In our study, we correlated ALL deficiency with functional limitation of activities; it was found that ACL deficient patients with ALL tear on MRI were able to do light activities only as compared to patients who had no ALL tear, who were able to do moderate and strenuous activities. After correlating patients with presence or absence of ALL tear on MRI with number of giving way episodes, we also found that patients with ALL tears had more episodes of giving way as compared with patients had no ALL tear, were highly associated. On comparing the ALL findings in MRI with pivot shift grading, it was found that ACL deficient knees with ALL tear showed higher grades of pivot shift phenomenon as compared to patients with ACL injury without ALL tear.

The timing between injury and MRI evaluation of ALL deficiency may have some significance. Hartigan et al. ${ }^{10}$ visualised the ALL on 1.5-T MRI in acute ACL injuries within three weeks in 72 patients. They reviewed the MRI for concomitant ALL tears, and visualized the ALL in $100 \%$ of the scans. They stated that previous to this study there was a mix of chronic and acute cases. Therefore, it could be suggested that the lower detection rate was related to chronicity of injury that had been evaluated, which probably confused the picture due to fibrosis of the ALL, as some element of repair could occur over time ${ }^{13}$. It is important to note that ALL injury detection is based on signal changes in acutely injured knees, which may be potentially reduced over a period of time. Therefore, it appears that factors such as the acute nature of the injury and timing of the MRI may be of some importance.

Prior controversy regarding the presence or absence of the ALL has been put to rest about its existence. 
Apart from our study, two previous studies in the literature have shown $100 \%$ visualization i.e. Hartigan et al. ${ }^{10}$ and Caterine et al. ${ }^{12}$. As in our study, only Hartigan et al. ${ }^{10}$ gave importance to the timing of MRI in relation of injury. We used high magnetic strength MRI i.e. 3T as compared to $1.5 \mathrm{~T}$ in previous studies which showed better resolution of images. Our study of Indian patients is the first to describe the radiological appearance of ALL in ACL deficient knees on $3 T$ $\mathrm{MRI}$ and correlate this with the clinical findings of the patients. Trinchese et al. ${ }^{17}$ have established a new technique of MIS anatomic reconstruction of the ALL, after identification by MRI, which further validates the role of this investigation.

Our study has some limitations: the sample size is somewhat small and lacks of validated scales for functional limitation of activities. It is to be emphasized that the radiologist has to be conversant with the anatomy of the ALL, so that special care may be taken to identify it. High resolution MRIs help, and a high index of suspicion has to be maintained in significantly unstable knee, to identify the ligament as well as the tear.

\section{Conclusion}

The ALL can be identified in all cases with 3T MRI. Tears are clearly seen and may even be classified according to tear location. We found no correlation of ALL tears with injury mechanism; however ACL deficient knees with concomitant ALL tear on MRI had more subjective functional impairment and instability. This ligament is thus an important stabiliser of the knee, and can be picked up by a trained radiologist using $3 \mathrm{~T} \mathrm{MRI}$, along with a high index of suspision, in the early weeks of injury, before a fibrotic healing response sets in and masks the MRI picture.

\section{Conflicts of interest}

None declared.

\section{References}

1. Goldman AB, Pavlov H, Rubenstein D. The Segond fracture of the proximal tibia: a small avulsion that reflects major ligamentous damage. Am J Roentgenol. 1988;151:1163-1167.

2. Dodds AL, Halewood C, Gupte CM, Williams A, Amis AA. The anterolateral ligament anatomy, length changes and associa- tion with the Segond fracture. Bone Joint J. 2014;96-B:325331.

3. Claes S, Bartholomeeusen S, Bellemans J. High prevalence of anterolateral ligament abnormalities in magnetic resonance images of anterior cruciate ligament-injured knees. Acta Orthop Belg. 2014;80:45-49.

4. Claes S, Vereecke E, Maes M, Victor J, Verdonk P, et al. Anatomy of the anterolateral ligament of the knee. J Anat. 2013;223:321-328.

5. Helito CP, Helder SM, Bonadio MB, Tirico LE, Gobbi RG, et al. Anatomical study on the anterolateral ligament of the knee. Rev Bras Ortop. 2013;48:368-373.

6. Assem "Mohamed Nour Eldin" Zein. Step-by-Step arthroscopic assessment of the anterolateral ligament of the knee using anatomic landmarks. Arthroscopy Techniques. 2015;4(6) e825-e831.

7. Beall DP, Googe JD, Moss JT, Greer BJ, Stapp AM, et al. Magnetic resonance imaging of the collateral ligaments and the anatomic quadrants of the knee. Radiol Clin North Am. 2007;45:983-1002

8. Helito CP, Helito PV, Costa HP, Rodrigues MB, Pecora JR, et al. MRI evaluation of the anterolateral ligament of the knee: assessment in routine 1.5-T scans. Skeletal Radiol. 2014;43: 1421-1427.

9. Padulo J, Oliva F, Frizziero A, Maffulli N. Muscles, Ligaments and Tendons Journal - Basic principles and recommendations in clinical and field science research: 2016 update. MLTJ. 2016;6(1):1-5.

10. Hartigan DE, Carroll KW, Kosarek FJ, Piasecki DP, Fleischli $\mathrm{JF}$, et al. Visibility of anterolateral ligament tears in anterior cruciate ligament deficient knees with standard 1.5-Tesla magnetic resonance imaging. Arthroscopy. 2016;32(10):20612065.

11. Jakob RP, Staubli HU and Deland JT. Grading the pivot shift objective tests with implications for treatment. J Bone Joint Surg Br. 1987;69(2)294-299.

12. Caterine S, Litchfield R, Johnson M, Chronik B, Getgood A. A cadaveric study of the anterolateral ligament: re-introducing the lateral capsular ligament. Knee Surg Sports Traumatol Arthrosc. 2015;23(11):3186-3195.

13. Kosy JD, Mandalia VI, Anaspure R. Characterization of the anatomy of the anterolateral ligament of the knee using magnetic resonance imaging. Skeletal Radiol. 2015;44(11):16471653.

14. Taneja AK, Miranda FC, Braga AP, Gill CM, Hartmann GC, et al. MRI features of the anterolateral ligament of the knee. Skeletal Radiol. 2015;44:411.

15. Helito CP, Demange MK, Helito PVP, Costa HP, Bonadio MB, et al. Evaluation of the anterolateral ligament of the knee by means of magnetic resonance examination. Rev Bras Ortop. 2015;50:214-219.

16. Chahla J, Menge TJ, Mitchell JJ, Dean CS, LaPrade RF. Anterolateral ligament reconstruction technique: An anatomicbased approach. Arthroscopy Techniques. 2016;5(3):e453 e457.

17. Trinchese GF, Oliva F, Maffulli N. Minimally invasive anatomic reconstruction of the anterolateral ligament with ipsilateral gracilis tendon. MLTJ. 2017;7(2):240-246. 